\title{
Adjuvante Chemotherapie des frühen operierten nicht-kleinzelligen Bronchialkarzinoms bringt Überlebensvorteil
} M. Serke

\author{
Adjuvant Chemotherapy in Non-Small-Cell Lung Cancer: Proven Survival Benefit
}

Obwohl eine britische Metaanalyse [1] zur Chemotherapie beim nicht-kleinzelligen Bronchialkarzinom NSCLC bereits 1995 einen $5 \%$ igen Überlebensvorteil nach einer platinhaltigen Chemotherapie ergab, konnten Studien zur adjuvanten Therapie des NSCLC bisher häufig keine positiven Ergebnisse zeigen.

So fand z.B. die ECOG-Studie [2] beim randomisierten Vergleich an 488 Patienten zwischen alleiniger Bestrahlung und simultaner Chemo-Strahlentherapie nach Operation von Patienten im Stadium II oder IIIA keinen Vorteil für die Chemo-Strahlentherapie-Gruppe. Kritisch anzumerken ist, dass die simultane ChemoStrahlentherapie trotz relativ niedriger Chemotherapiedosis (Cisplatin: $1 \times 60 \mathrm{mg} / \mathrm{m}^{2}$, Etoposid: $3 \times 120 \mathrm{mg} / \mathrm{m}^{2}$, Wiederholung Tag 28) zu erheblicher Toxizität führte, weshalb die Chemotherapie bei nur 69\% der Patienten abgeschlossen werden konnte. Insgesamt war die Chemotherapie offenbar wegen der zu geringen Dosisintensität nicht effektiv. Lediglich bei einer Subgruppe (Frauen mit Nicht-Plattenepithelkarzinom) konnte im Chemotherapie-Arm ein signifikant besseres Überleben (ÜL) (medianes ÜL 25 Monate vs. 43 Monate, $p=0,002$ ) nachgewiesen werden.

Eine weitere große internationale Studie (ALPI-EORTC) [3] zum Vergleich einer adjuvanten Chemotherapie des NSCLC nach kurativer Resektion versus Beobachtung wurde vorzeitig abgebrochen. Sie konnte in den Stadien I - IIIA nach Einschluss von 1200 Patienten unter 3 Zyklen adäquat dosierter Chemotherapie (Mito (M): $8 \mathrm{mg} / \mathrm{m}^{2}$, VDS (V): $3 \mathrm{mg} / \mathrm{m}^{2} \mathrm{~d} 1+8 /$ Cisplatin (P): $100 \mathrm{mg} / \mathrm{m}^{2}$ d1, MVP, Wiederholung Tag 21) keinen signifikanten Vorteil für die Chemotherapie-Gruppe zeigen. Die Überlebenskurven in der Kaplan-Meier-Darstellung verliefen praktisch parallel, Ausdruck der fehlenden Überlebensverbesserung, obwohl in der Chemo-
therapie-Gruppe etwas weniger Rezidive als in der KontrollGruppe beobachtet wurden (195 vs. 234). Offenbar war auch in dieser Studie die erreichte Dosisintensität der Chemotherapie nicht wirksam genug und die Toxizität der angebotenen adjuvanten Chemotherapie zu hoch.

Ein erstes positives Ergebnis konnte mit einer kombinierten präund postoperativen Chemotherapie (neoadjuvant und adjuvant) mit Mitomycin/Ifosfamid/Cisplatin (MIC) [4] erreicht werden. Hier konnte beim Vergleich zwischen prä- und postoperativer sequentieller Chemo-Strahlentherapie im Vergleich zur alleinigen OP und Bestrahlung ein signifikanter Vorteil zugunsten der Chemotherapie-Gruppe beobachtet werden. Das Überleben war in der Chemotherapie-Gruppe verlängert und das Metastasierungsrisiko war signifikant verringert, obwohl die Therapieassoziierte Mortalität im Chemotherapie-OP-Bestrahlungs-Arm gegenüber dem alleinigen $\mathrm{OP}$ - und Bestrahlungs-Arm verdoppelt war ( $10 \%$ vs. $4,5 \%)$.

Nachdem eine erste japanische Studie einer adjuvanten Chemotherapie mit dem oralen Antimetaboliten Uracil-Tegafur (UFT), der als sog. „prodrug“ letztlich zu Fluorouracil metabolisiert wird, einen Überlebensvorteil für die mit UFT langfristig über 2 Jahre behandelten Patienten ergab, konnte eine zweite große randomisierte Studie an 999 Patienten dies für T2-Adenokarzinome im Stadium I (T2N0) bestätigen [5]. Während sich im T1-Stadium (T1N0) kein Überlebensvorteil unter der adjuvanten Chemotherapie nachweisen ließ, war das 5-Jahresüberleben bei den größeren T2-Tumoren mit $85 \%$ versus $74 \%$ signifikant verbessert, obwohl auch hier die Compliance nicht optimal war: Nur $61 \%$ der Patienten nahmen die Medikation über die vorgesehenen zwei Jahre lang ein. 
IALT [6] nun ist ein Meilenstein in der adjuvanten Therapie des NSCLC. Es handelt sich um die bisher größte randomisierte Studie mit 1867 Patienten zur adjuvanten Therapie des frühen operierten NSCLC der Stadien I-III. Operierte Patienten erhielten 3-4 Zyklen einer cisplatinbasierten Therapie (mit frei gewähltem zweitem Kombinationspartner der 2. Generation: Etoposid oder Vinca-Alkaloid) in einem „open-choice-design“. IALT konnte ein statistisch signifikantes positives Ergebnis zugunsten der adjuvanten Therapie zeigen: Das 5-Jahres-rezidivfreie Überleben war in der Chemotherapie-Gruppe mit 39,4\% gegenüber 34,3\% in der Beobachtungsgruppe deutlich günstiger, auch das 5-Jahresüberleben war in der Chemotherapie-Gruppe mit 44,5\% dem in der Kontrollgruppe mit 40,4\% signifikant überlegen.

Innerhalb der Studienpopulation ergaben sich keine Unterschiede der Wirksamkeit innerhalb der Stadien (I-III) oder der Morphologie, so dass die adjuvante Chemotherapie für alle Subgruppen von Vorteil zu sein verspricht.

IALT zeigt somit, dass auch beim nicht-kleinzelligen Bronchialkarzinom durch die adjuvante Therapie ein Überlebensvorteil erreicht wird, wie es zuvor schon bei anderen soliden Tumoren wie dem Mamma-, Kolon oder Magenkarzinom gezeigt wurde.

Die adjuvante Chemotherapie beim NSCLC verspricht eine „moderate“ Überlebensverbesserung, entsprechend einem um $4 \%$ verbesserten 5 -Jahresüberleben und einem um $5 \%$ verbesserten rezidivfreien 5-Jahres-Überleben.

Im IALT-Protokoll war, um den Einschluss zu erleichtern, der Chemotherapie-Kombinationspartner zum Cisplatin nicht festgelegt, daher kann das Studienergebnis für alle beim NSCLC wirksamen cisplatinhaltigen Standard-Regime übernommen werden, ohne dass eine Festlegung auf eine einzelne Chemotherapie-Kombination notwendig ist.

Allerdings macht diese Studie auch deutlich, dass noch Probleme mit der Toxizität der Therapie und der Compliance zu beachten sind. Auch bei IALT war, wie häufig in Studien zur adjuvanten Therapie des NSCLC beobachtet, die letztlich verabreichte Dosis deutlich niedriger als die geplante: Insgesamt wurden nur ca. $74 \%$ der ursprünglich geplanten Chemotherapie-Dosis gegeben und fast ein Zehntel (8\%) der für die Chemotherapie randomisierten Patienten erhielten gar keine Chemotherapie. Dennoch wurde eine nicht akzeptable Chemotherapie-bedingte Mortalität von $0,8 \%$ beobachtet und eine nicht unerhebliche Toxizität der Therapie. Sie führte bei einem Viertel der Patienten $(22,6 \%) \mathrm{zu}$ mindestens einer Episode mit lebensbedrohlicher Toxizität, meist in Form von Neutropenie (17,5\%), Thrombopenie (2,6\%) oder intensivem Erbrechen (3,3\%), so dass in dieser Hinsicht Verbesserungen in der Therapie wünschenswert sind.

Wesentliche Voraussetzungen für eine umfassende zukünftige Anwendung einer adjuvanten Therapie werden eine vernünftige und effektive Dosierung bei möglichst geringer Toxizität sein. Möglicherweise kann dies durch die Verwendung von Zytostatika der dritten Generation mit verringerter Toxizität und damit Vermeidung von Therapieabbrüchen und Dosisreduktionen erreicht werden. Günstig wäre es auch, wenn weitere Bestätigungsstudien, z.B. mit Zytostatika der 3. Generation, folgen könnten.

Als praktische Konsequenz ergibt sich:

Die adjuvante Therapie beim NSCLC

- ist ähnlich effektiv wie bei anderen soliden Tumoren mit einem um $4 \%$ verbesserten 5 -Jahresüberleben

- ist zur Zeit mit Compliance- und Toxizitätsproblemen belastet

- kann möglicherweise mit den NSCLC-Medikamenten der 3. Generation oder durch Ersatz von Cisplatin durch Carboplatin verträglicher gestaltet werden

- sollte möglichst im Rahmen von klinischen Studien angewandt und optimiert werden

- kann bei gutem Allgemeinzustand und fehlenden Begleiterkrankungen als individuelle Entscheidung auch außerhalb von Studien eingesetzt werden.

\section{Literatur}

${ }^{1}$ Non-small cell lung cancer collaborative group. Chemotherapy in nonsmall cell lung cancer: a meta-analysis using updated data on individual patients from 52 randomized clinical trials. BMJ 1995; 311: 899-909

${ }^{2}$ Keller SM, Adak S, Wagner $\mathrm{H}$ et al. A randomized trial of postoperative adjuvant therapy in patients with completely resected stage II or IIIA non-small-cell lung cancer. N Eng J Med. 2000; 343: 1217-1222

${ }^{3}$ Scagiotti GV, Fossati R, Torri V et al. Randomized study of adjuvant chemotherapy for completely resected stage I, II or IIIA non-small-cell lung cancer. J Natl Cancer Inst 2003; 95: 1453-1461

${ }^{4}$ Depierre A, Milleron B, Moro-Sibilot D et al. Preoperative chemotherapy followed by surgery compared with primary surgery in respectable stage I (except T1N0), II, and IIIa non-small cell lung cancer. J Clin Oncol 2002; 20: 247-253

${ }^{5}$ Kato $\mathrm{H}$, Ichinose $\mathrm{Y}$, Ohta $\mathrm{M}$ et al. A randomized trial of adjuvant chemotherapy with uracil-Tegafur for adenocarcinoma of the lung. $\mathrm{N}$ Eng J Med 2004; 350: 1713-1721

${ }^{6}$ The International Adjuvant Lung Cancer Collaborative Group. Cisplatin-based adjuvant Chemotherapy in patients with completely resected Non-Small-Cell Lung Cancer. N Eng J Med 2004; 350: 351 - 360 